Monatshefte für Chemie 107, 449-458 (1976)

(c) by Springer-Verlag 1976

\title{
Sila-Analogon des Cicloniumbromids
}

\author{
Sila-Pharmaka, 4. Mitt. ${ }^{1}$ \\ Von \\ Reinhold Tacke ${ }^{2}$ und Ulrich Wannagat \\ Institut für Anorganische Chemie, \\ Technische Universität Braunschweig, Bundesrepublik Deutschland
}

(Eingegangen am 24. September 1975)

Sila-Analogue of Ciclonium Bromide

Sila-Analogues B 2 and A 2 of the spasmolytic ciclonium bromide (B 1) respectively the corresponding free base A 1 were synthesized for the first time according to the reaction steps shown in scheme 1, and they and their precursors I and II were characterized by physical (Table 1) and chemical properties and their structures confirmed by NMR, and mass spectroscopy (Tables 2 and 3 ). The pharmacological effects of A 2 and B 2 were investigated and compared with those of the parent carbon compound B 1 (chapter 5).

\section{Einführung}

Zur therapeutischen Beeinflussung spastischer Zustände der glatten Muskulatur hat sich das Spasmolytikum Cicloniumbromid (B 1) sehr gut bewährt. Dieses Pharmakon ist auf breiter Basis verwendbar, da es dosisabhängig sowohl eine starke parasympatholytische als auch muskulotrop spasmolytische Wirkkomponente besitzt ${ }^{3}$. Das Cicloniumbromid steht als quartäre Ammoniumverbindung den Antihistaminika

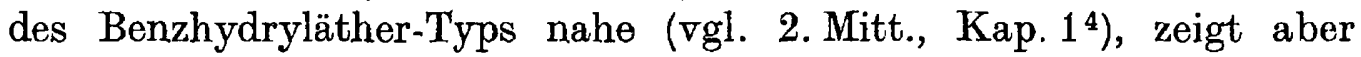
auf Grund gewisser Strukturmerkmale stark veränderte pharmakologische Eigenschaften ${ }^{3}$. Die Substitution einer Phenylgruppe durch den Bicyclus und die Überführung der tertiären Aminogruppe in eine quartäre Ammoniumgruppe ergeben eine starke Abnahme der Antihistaminwirkung, während die cholinolytische Wirkkomponente beträchtlich zunimmt. Des weiteren ist eine Passage der Blut-HirnSchranke auf Grund der Ammoniumstruktur sehr stark erschwert, so daß das Cicloniumbromid im Gegensatz zu den BenzhydrylätherAntihistaminika praktisch keinerlei zentrale Wirkungen entfalten kann. 
In Fortführung unserer Untersuchungen über Sila-Pharmaka, bei denen wir uns bisher mit Sila-Analoga der Benzhydryläther-Antihistaminika (vgl. 1. Mitt. ${ }^{5}, 2$. Mitt. ${ }^{4}, 3$. Mitt. ${ }^{1}$ ) beschäftigten, stellten wir ein Sila-Cicloniumbromid (,Si-Adamon“, B 2) her, über dessen Synthese und Eigenschaften im folgenden berichtet werden soll.

\section{Zur Darstellung des Sila-Cicloniumbromids}

Das Cicloniumbromid (B 1) wurde, von $\alpha$-(Bicyclo[2.2.1] hept-2-en-5-y1)$\alpha$-methyl-benzylalkohol ausgehend, durch Metallierung mit Natriumamid, Umsetzung mit 1-Chlor-2-diäthylaminoäthan und anschließende Quaternierung mit Methylbromid dargestellt ${ }^{6}$ (Skizze 1).

Die Synthese des Sila-Analogons konnte auf folgendem Wege realisiert werden: Die Diels-Alder-Reaktion von Vinyl-trichlorsilan mit Cyclopentadien führt über Rk. (1) zum bereits bekannten (Bicyclo[2.2.1]hept-2-en-5-yl)-trichlorsilan (I), das sich durch Grignardierung mit Phenylmagnesiumbromid (Rk.2) in das bisher unbekannte (Bicyclo[2.2.1]hept-2-en-5-yl)-phenyldichlorsilan (II) und durch erneute Grignardierung mit Methylmagnesiumjodid (Rk.3) in das ebenfalls neue (Bicyclo[2.2.1]hept-2-en-5-yl)-methyl-phenyl-chlorsilan (III) überführen läßt. Letzteres reagiert — es wurde als Rohprodukt eingesetzt, da die Verbindung destillativ nicht rein isoliert werden konnte - mit 2-Diäthylaminoäthanol gemäß Rk. (4) zu A 2, das durch Umsetzung mit Methylbromid [Rk. (5)] schließlich das Sila-Cicloniumbromid (B 2) ergibt.

\section{Experimenteller Teil}

(Bicyclo[2.2.1]hept-2-en-5-yl)-trichlorsilan (I): [Rk. (1)]. In Anlehnung an 7 durch vorsichtiges 3stdg. Erwärmen von $273 \mathrm{~g}$ (1,69 Mol) Vinyl-trichlorsilan mit $95 \mathrm{~g}(1,44 \mathrm{Mol})$ frisch destill. Cyclopentadien auf max. $80^{\circ}$ und kurzzeitiges Erhitzen auf $120^{\circ} \mathrm{C}$. Fraktionierende Destillation im Vak. über eine Vigreux-Kolonne liefert $250 \mathrm{~g} \mathrm{I}$.

Die im Rahmen der vorliegenden Arbeit durchgeführten Untersuchungen und die weiteren Umsetzungen dieser Substanz beziehen sich alle auf das Gemisch aus endo- und exo-Addukt ${ }^{8-10}$. Eine quantitative Anteilsbestimmung der beiden Isomeren wurde von uns nicht durchgeführt.

(Bicyclo[2.2.1] hept-2-en-5-yl)-phenyl-dichlorsilan (II): [Rk. (2)]. Man tropft bei $20^{\circ} \mathrm{C}$ innerhalb $2 \mathrm{Stdn}$. unter starkem Rühren eine aus $12,4 \mathrm{~g}$ $(0,51 \mathrm{Mol}) \mathrm{Mg}$ und $78,5 \mathrm{~g}(0,5 \mathrm{Mol})$ Brombenzol in $400 \mathrm{ml}$ Diäthyläther (= $\ddot{A})$ bereitete Grignard-Lösung zu $113,8 \mathrm{~g}(0,5 \mathrm{Mol}) \mathrm{I}$ in $250 \mathrm{ml} \ddot{A}$, rührt 1 Stde. unter Rückfluß weiter, tropft in der Hitze $100 \mathrm{ml} T H F$ hinzu, rührt $12 \mathrm{Stdn}$. bei $20^{\circ} \mathrm{C}$ weiter, filtriert, wäscht dreimal mit je $50 \mathrm{ml}$ Petroläther $\left(50 / 70^{\circ} \mathrm{C}\right) \quad(=P \ddot{A})$ nach, filtriert erneut, zieht das Lösungsmittel von den vereinigten Filtraten ab, nimmt den Rückstand in $300 \mathrm{ml} P \ddot{A}$ auf, filtriert über $\mathrm{Na}_{2} \mathrm{SO}_{4}{ }^{5}$, zieht den $P \ddot{A}$ ab und destilliert fraktionierend im Vak. über eine Vigreux-Kolonne; Ausb. 60,6 g II. 
Sila-Analogon des Cicloniumbromids
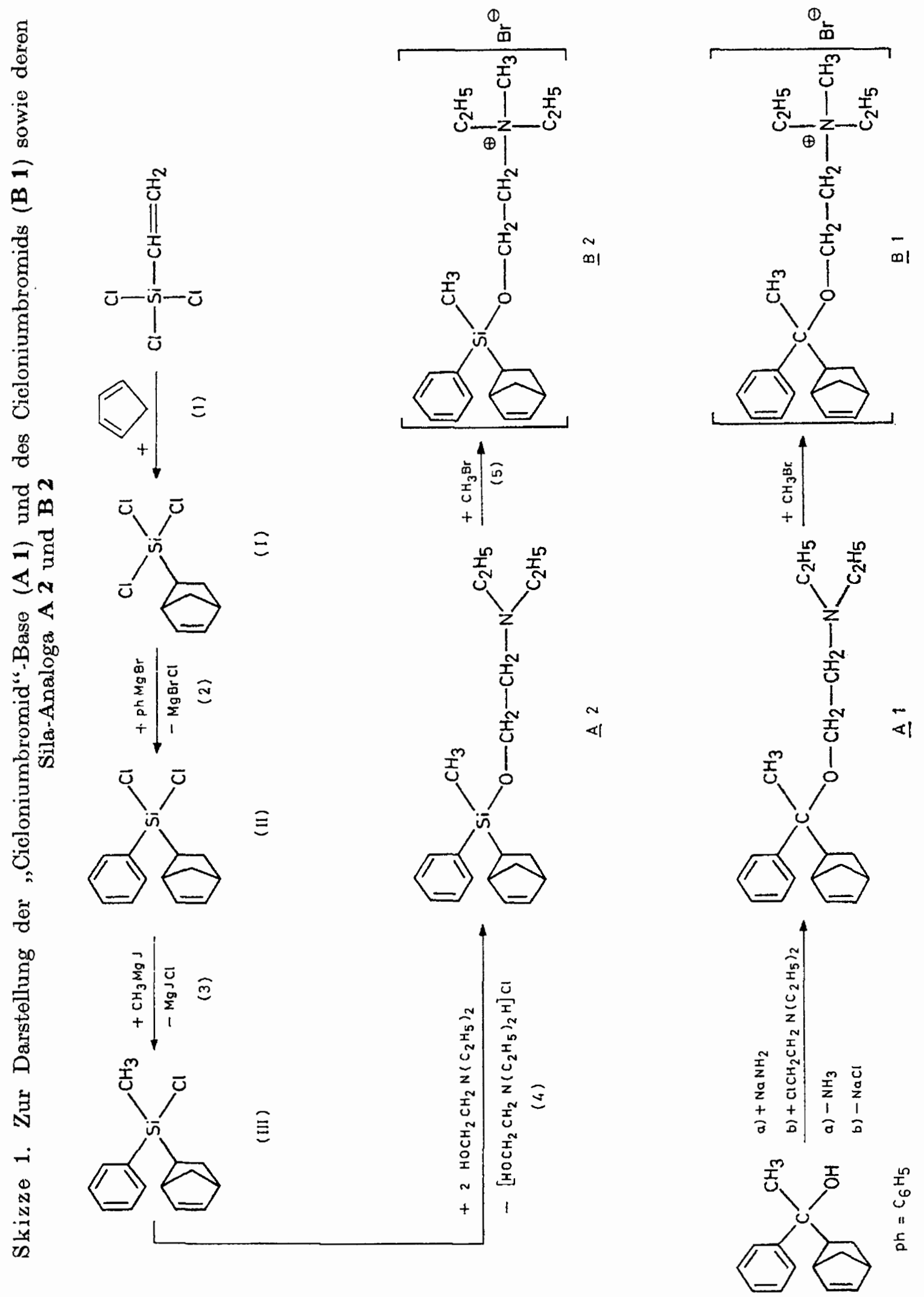
(Bicyclo[2.2.1]hept-2-en-5-yl)-(methyl)-phenyl-chlorsilan (III): [Rk. (3)]. Analog zu Rk. (2) durch 2stdg. Zutropfen einer aus $7,3 \mathrm{~g}(0,3 \mathrm{Mol}) \mathrm{Mg}$ und $42,6 \mathrm{~g}(0,3 \mathrm{Mol}) \mathrm{CH}_{3} J$ in $250 \mathrm{ml} \ddot{A}$ bereiteten Grignard-Lösung zu $80 \mathrm{~g}(0,3 \mathrm{Mol})$ II in $200 \mathrm{ml} \ddot{A}$ und analoge Aufarbeitung $(80 \mathrm{ml} T H F$, $3 \mathrm{mal}$ je $40 \mathrm{~mol} P \ddot{A}, 250 \mathrm{ml} P \ddot{A}$ ).

Bei der Destillation über eine Vigreux-Kolonne erhält man etwa $60 \mathrm{~g}$ einer Fraktion im Siedeintervall von $87-100^{\circ} \mathrm{C}(0,01$ Torr $)$. Das gewünschte (monomethyl-substituierte) III ist dabei hauptsächlich durch das Ausgangsprodukt (II) und die entsprechende dimethyl-substituierte Verbindung verunreinigt. Da III nicht rein isoliert werden konnte, wurde es als Rohprodukt weiter umgesetzt ( $\rightarrow$ Rk. 4).

$N, N$-Diäthyl-2-[(bicyclo[2.2.1]hept - 2 - en - 5 - yl) - methyl-phenylsilyloxy] äthylamin (A 2): [Rk. (4)]. Man tropft zu 210,9 g (1,8 Mol) 2-Diäthylamino$\ddot{a}$ thanol in $600 \mathrm{ml} P \ddot{A}$ langsam und unter starkem Rühren eine Lösung von $60 \mathrm{~g}$ III (als Rohprodukt) in $450 \mathrm{ml} P \ddot{A}$, wobei sofort 2-Diäthylaminoäthanolhydrochlorid ausfällt, rührt 1 Stde. bei $20^{\circ} \mathrm{C}, 4$ Stdn. unter Rückfluß weiter, filtriert vom Hydrochlorid, wäscht es mit $100 \mathrm{ml} P \ddot{A}$, zieht das Lösungsmittel ab und fraktioniert den Rückstand im Ölpumpenvak. 3mal über eine Vigreux-Kolonne. Ausb. $34 \mathrm{~g}$ A 2 [34,7\% für Rkk. (3) und (4), bezogen auf $0,3 \mathrm{Mol}$ II].

$N, N$-Diäthyl-N-methyl-2-[(bicyclo[2.2.1]hept-2-en - 5-yl)-methyl-phenyl silyloxy]-äthylammoniumbromid (B 2): [Rk. (5)]. Man gibt unter Rühren zu 3,3 g (0,01 Mol) A 2 in $30 \mathrm{ml}$ Acetonitril eine Lösung von 5,7 g $(0,06 \mathrm{Mol})$ $\mathrm{CH}_{3} \mathrm{Br}$ in $40 \mathrm{ml}$ Acetonitril, rührt 1 Stde. bei $20^{\circ} \mathrm{C}$, kocht $1 \frac{1}{2} \mathrm{Stdn}$. unter schwachem Rückfluß, läßt auf $20^{\circ} \mathrm{C}$ abkühlen, dampft im Ölpumpenvak. ab, nimmt den Rückstand in $2 \mathrm{ml}$ Acetonitril auf, gibt etwa $0,2 \mathrm{ml} P \ddot{A}$ hinzu und läßt solange bei $-20^{\circ} \mathrm{C}$ stehen, bis B 2 auskristallisiert ist. Nach zweimaligem Umkristallisieren aus einem Acetonitril- $P \ddot{A}$-Gemisch $(10: 1)$ und anschließendem Trocknen der Kristalle (5 Stdn. bei $20^{\circ} \mathrm{C}$ ) im Hochvak. fällt B 2 in analysenreiner Form an. Ausb. 2,6--2,9 g B 2.

3. Chemische und physikalische Eigenschaften des SilaCicloniumbromids, der freien Base A 2 sowie der Vorstufen I und II

Die Vorstufen I, II und III sind wasserklare hydrolyseempfindliche Flüssigkeiten, die an der Luft rauchen. Sie lösen sich gut in den üblichen inerten organischen Lösungsmitteln und sind unter vermindertem Druck unzersetzt destillierbar (Tab. 1).

Die von uns ermittelten physikalischen Daten von I (als IsomerenGemisch) stimmen weitgehend mit den Literaturangaben 7,8 überein Die entsprechenden Daten von II (als Isomerengemisch) liegen im Erwartungsbereich. III konnte nicht rein isoliert werden (vgl. Kap. 2).

A 2, die freie Base des Sila-Cicloniumbromids, ist eine wasserklare, ölige Flüssigkeit, die sich unzersetzt im Vak. destillieren läßt. Sie löst sich gut in den gängigen organischen Solventien, schlecht dagegen in 


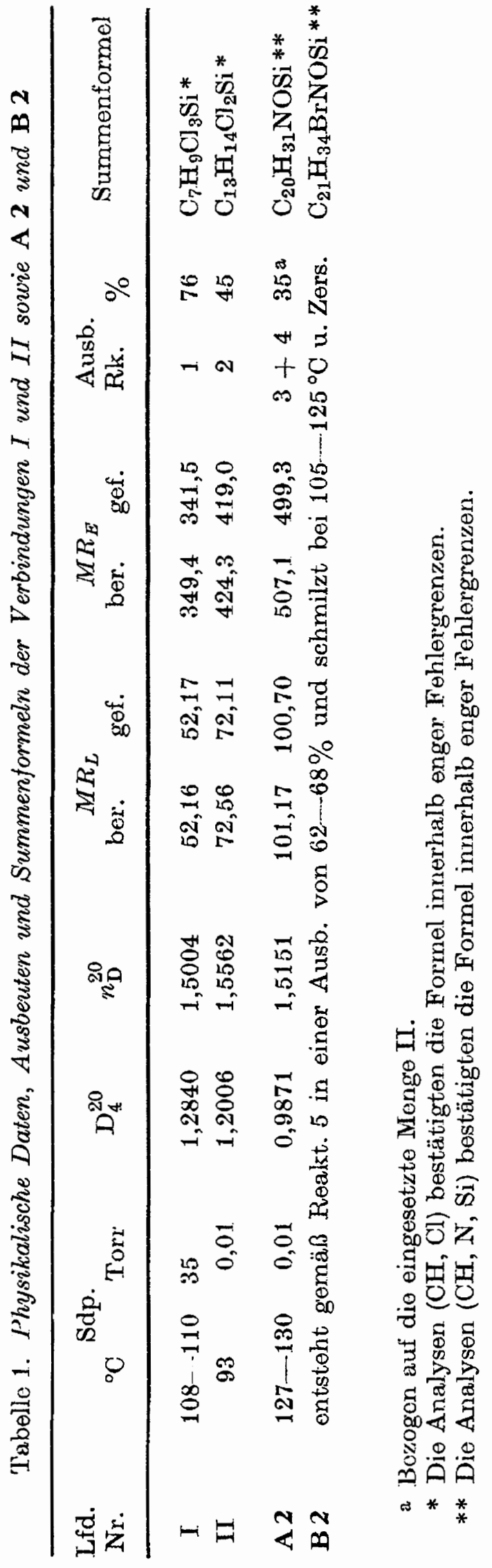


Wasser. Es kommt zur Ausbildung eines Zweiphasensystems, an dessen Grenzfläche Hydrolysereaktionen (Spaltung der Si-OC-Gruppierung) ablaufen. Bei guter Verteilung und einer kleinen Konzentration $\left(10^{-6} \mathrm{Mol} / \mathrm{l}\right)$ von A 2 in Tyrode-Lösung (Wasser, gepuffert auf $\mathrm{pH} 7,4 ; 37^{\circ} \mathrm{C}$ ) erfolgt der hydrolytische Abbau von A 2 in etwa $28 \mathrm{Min}$., wie sich an Hand von pharmakologischen Messungen erkennen ließ (vgl. Kap. 5).

Tabelle 2. ${ }^{1} \mathrm{H}-\mathrm{NMR}$-Spektren der Verbindungen $I$ und $I I$ sowie A 2 und B 2. Chemische Verschiebungen in $\delta$ [ppm], relative Intensitäten in Klammern, Lösungsmittel $\mathrm{CCl}_{4}$ (bei I ohne Lösungsmittel, bei $\mathrm{B} 2 \mathrm{CDCl}_{3}$ ); Lock intern: $T M S$

\begin{tabular}{ccccl}
\hline $\begin{array}{c}\text { Lfd. } \\
\text { Nr. }\end{array}$ & $\begin{array}{c}\text { aromatische } \\
\text { Protonen }\end{array}$ & $\begin{array}{c}\text { olefinische } \\
\text { Protonen }\end{array}$ & $\begin{array}{c}\text { Brückenkopf- } \\
\text { Protonen }\end{array}$ & $\begin{array}{c}\text { übrige } \\
\text { Protonen }\end{array}$ \\
\hline I & & & & \\
II & $7,9-7,1(5)$ & $6,3-5,8(2)$ & $3,4-2,9(2)$ & $2,4-1,0(5)$ \\
A2 & $7,8-7,0(5)$ & $6,2-5,7(2)$ & $3,3-2,7(2)$ & $2,3-1,0(5)$ \\
B2 & $7,8-7,0(5)$ & $6,3-5,7(2)$ & & $3,3-0,2(24)^{\mathrm{a}, \mathrm{b}, \mathrm{c}}$ \\
& & & $4,4-0,2(27)^{\mathrm{a}, \mathrm{d}, \mathrm{e}, \mathrm{f}}$
\end{tabular}

a Einschließlich Brückenkopf-Protonen.

b Im Erwartungsbereich für $\mathrm{Si}-\mathrm{CH}_{3}$-Protonen zwei etwa gleich starke Singuletts bei 0,40 und 0,25 (Hinweis auf mindestens zwei diastereomere Formen).

c Für die $\mathrm{N}-\mathrm{C}_{2} \mathrm{H}_{5}$-Protonen bei 0,97 ein Triplett und bei 2,48 ein Quartett mit der Kopplungskonstanten $J_{\text {vic }} \mathrm{H}-\mathrm{C}-\mathrm{C}-\mathrm{H}=7 \mathrm{~Hz}$.

d Im Erwartungsbereich für $\mathrm{Si}-\mathrm{CH}_{3}$-Protonen zwei etwa gleich starke Singuletts bei 0,47 und 0,32 (Hinweis auf mindestens zwei diastereomere Formen).

e Für die $\stackrel{\oplus}{\mathrm{N}}-\mathrm{C}_{2} \mathrm{H}_{5}$-Protonen bei 1,39 ein Triplett und bei 3,68 ein Quartett mit der Kopplungskonstanten $J_{\text {vic }} \mathrm{H}-\mathrm{C}-\mathrm{C}-\mathrm{H}=7 \mathrm{~Hz}$.

f Für die $\stackrel{\oplus}{\mathrm{N}}-\mathrm{CH}_{3}$-Protonen bei 3,27 ein Singulett.

Auf Grund der gegebenen stereochemischen Voraussetzungen kann A 2 maximal als Gemisch von vier zueinander diastereomeren Enantiomerenpaaren vorliegen. Über das tatsächliche Vorhandensein und den mengenmäßigen Anteil der einzelnen Isomeren können wir allerdings keine Aussagen machen. Die Existenz zweier etwa gleich starker $\mathrm{Si}-\mathrm{CH}_{3}$-Signale im ${ }^{1} \mathrm{H}-\mathrm{NMR}-\mathrm{Spektrum}$ (siehe Tab.2) von A 2 läßt lediglich auf das Vorhandensein von mindestens zwei Enantiomerenpæaren schließen. Eine destillative Trennung gelang erwartungsgemäß nicht, so daß sich alle an A 2 durchgeführten Untersuchungen auf das Isomerengemisch beziehen.

Das Sila-Cicloniumbromid ist eine weiße, kristalline Substanz, 
die im Intervall von etwa $105-125^{\circ} \mathrm{C}$ unter Zersetzung schmilzt. Sie löst sich gut in Äthanol, $\mathrm{CHCl}_{3}$ und $\mathrm{CH}_{3} \mathrm{CN}$, nicht aber in $\mathrm{CCl}_{4}$, $\mathrm{C}_{6} \mathrm{H}_{6}$ und Diäthyläther. Die Löslichkeit in Wasser ist sehr gut; die völlig klare wäßrige Lösung wird allerdings nach etwa 1 Min. deutlich trübe, da B 2 hydrolysiert. Das Sila-Cicloniumbromid liegt ebenfalls als Isomerengemisch - es sind acht Isomere denkbar - vor.

Tabelle 3. m/e-Werte der Massenspektren der Verbindungen $I, I I$ und A 2 mit Molpeaks $M^{+}$sowie einigen charakteristischen Schlüsselbruchstücken. Angabe der relativen Intensitäten (Basispeak $=100$ ) und Chlorisotopenmuster. ElektronenstoBionisierung mit $70 \mathrm{eV}$. Probeneinführung durch Direkteinlaß

\begin{tabular}{|c|c|c|c|c|}
\hline $\begin{array}{l}\text { Lfd. } \\
\text { Nr. }\end{array}$ & Fragmente & $m / e$-Werte & $\begin{array}{l}\text { Chlorisotopen- } \\
\text { muster }\end{array}$ & $\begin{array}{c}\text { relative } \\
\text { Intensitäten }\end{array}$ \\
\hline I & $\begin{array}{l}M^{+} \\
M^{+}-(\mathrm{R})^{\mathrm{a}} \\
\mathrm{C}_{5} \mathrm{H}_{6} 7^{+}\end{array}$ & $\begin{array}{l}226 / 228 / 230 / 232 \\
133 / 135 / 137 / 139 \\
66\end{array}$ & $\begin{array}{l}\mathrm{Cl}_{3} \\
\mathrm{Cl}_{3}\end{array}$ & $\begin{array}{l}<1 \\
11 / 11 / 4 /<1 \\
100\end{array}$ \\
\hline II & $\begin{array}{l}M^{+} \\
M^{+}-(\mathrm{R})^{a} \\
\mathrm{C}_{6} \mathrm{H}_{5} 7^{+} \\
\mathrm{C}_{3} \mathrm{H}_{6} 7^{+}\end{array}$ & $\begin{array}{l}268 / 270 / 272 \\
175 / 177 / 179 \\
77 \\
66\end{array}$ & $\begin{array}{l}\mathrm{Cl}_{2} \\
\mathrm{Cl}_{2}\end{array}$ & $\begin{array}{l}3 / 2 /<1 \\
16 / 11 / 2 \\
19 \\
100\end{array}$ \\
\hline A 2 & $\begin{array}{l}M^{+} \\
M^{+}-\left(\mathrm{CH}_{3}\right) \\
M^{+}-(\mathrm{R})^{\mathrm{a}} \\
\mathrm{SiR}^{+\mathrm{a}} \\
\left.\mathrm{SiC}_{6} \mathrm{H}_{5}\right]^{+} \\
\left.\mathrm{CH}_{2}=\mathrm{N}\left(\mathrm{C}_{2} \mathrm{H}_{5}\right)_{2}\right\rceil^{+} \\
\mathrm{C}_{6} \mathrm{H}_{5} 7^{+} \\
\left.\mathrm{C}_{5} \mathrm{H}_{6}\right\rceil^{+} \\
\left.\mathrm{CH}_{2}=\dot{\mathrm{N}}\left(\mathrm{C}_{2} \mathrm{H}_{5}\right) \mathrm{H}\right\rceil^{+}\end{array}$ & $\begin{array}{r}329 \\
314 \\
236 \\
121 \\
105 \\
86 \\
77 \\
66 \\
58\end{array}$ & & $\begin{array}{r}5 \\
1 \\
5 \\
10 \\
2 \\
100 \\
1 \\
5 \\
6\end{array}$ \\
\hline
\end{tabular}

4. Zur Strukturermittlung des Sila-Cicloniumbromids

der freien Base A 2 sowie ihrer Vorstufen I und II wurden neben den Elementaranalysen vor allem ${ }^{1}$ H-NMR-Spektren und, mit Ausnahme von B 2, Massenspektren herangezogen.

Die Protonenresonanzspektren (siehe Tab. 2) erwiesen sich für den Strukturbeweis als sehr nützlich. Da alle Verbindungen als Isomerengemische vorlagen und die einzelnen Protonen sehr komplizierte Aufspaltungsmuster zeigen, ist eine exakte Interpretation und vollständige Zuordnung der Resonanzsignale nicht möglich. Man findet 
in den Spektren jedoch typische Signalgruppen mit charakteristischen Bereichen der chemischen Verschiebung, so für den Bicyclus: olefinische Protonen bei $\delta=5,7-6,3 \mathrm{ppm}$, Brückenkopf-Protonen bei $\delta=2,7-3,4 \mathrm{ppm}$ und übrige Protonen bei $\delta=1,0-2,4 \mathrm{ppm}$. Die aromatischen Protonen finden sich weitgestreut als Multiplett im Bereich $\delta=7,0-7,9 \mathrm{ppm}$. Zusätzlich $\mathrm{zu}$ diesen charakteristischen Signalgruppen ist für einige Resonanzen eine exakte Zuordnung möglich (siehe Tab. 2).

Die Massenspektren (siehe Tab. 3) lassen für die Verbindungen I und II den Molpeak mit den charakteristischen Chlor-Isotopenmustern erkennen. Für beide Verbindungen findet man den Basispeak bei $m / e=66$. Möglicherweise handelt es sich hier um ein $\mathrm{C}_{5} \mathbf{H}_{6}{ }^{{ }^{\oplus}}$-Ion, das aus einem Retro-Diels-Alder-Zerfall hervorgegangen ist. Im Massenspektrum von A 2 finden sich ebenfalls das Molekülion sowie einige charakteristische Schlüsselbruchstücke, die strukturbeweisend sind. Hierzu analoge Fragmente wurden schon früher bei der massenspektroskopischen Untersuchung von Silabenzhydryläthern gefunden 1, 4, 5 . Ebenso wie diese Substanzklasse liefert auch A 2 mit dem $\mathrm{CH}_{2}=\stackrel{\oplus}{\mathrm{N}}\left(\mathrm{C}_{2} \mathrm{H}_{5}\right)_{2}$-Ion den Basispeak.

5. Pharmakologische und toxikologische Eigenschaften der Sila-Pharmaka A 2 und B 2

Die pharmakologischen und toxikologischen Untersuchungen wurden in enger Zusammenarbeit am Institut für Pharmakologie und Toxikologie der Technischen Universität Braunschweig von F. Meyer und H.-U. Rossée durchgeführt. Für ausführliche Informationen sei auf die Dissertation Rossée ${ }^{11}$ verwiesen.

Die beiden Sila-Pharmaka A 2 und B 2 sind pharmakodynamisch wirksam als kompetitive Antagonisten der Spasmodika Histamin und Carbachol und vermögen außerdem einen durch Bariumionen erzeugten Spasmus abzubauen. Untersuchungen am isolierten Meerschweinchen-Ileum zeigen sehr deutlich, daß die Substitution einer Phenylgruppe durch eine Norbornenylgruppe im Strukturgerüst der Benzhydryläther-Antihistaminika zu pharmakologischen Eigenschaften führt, die sich von denen der Antihistaminika erheblich unterscheiden. So ergibt der Vergleich der $E D_{50}$-Werte ${ }^{11}$ von A 2 mit denen des SilaMephenhydramins ${ }^{5}$, Sila-Chlorphenoxamins ${ }^{4}$ und Sila-Clofenetamins ${ }^{4}$, daß diese Substitution einen deutlichen Verlust an histaminolytischer Aktivität und einen sehr starken Gewinn an anticholinerger Wirkung verursacht. Die Quaternierung von A 2 führt zu einer weiteren Spezi- 
fitätserhöhung cholinolytischer Aktivität, wie der Wirkungsvergleich mit B 2 ergibt.

Das Sila-Cicloniumbromid B 2 ist sowohl gegen Carbachol als auch gegen Bariumionen praktisch gleich wirksam wie die C-Muttersubstanz B 1. Dagegen ist die Silicium-Verbindung gegen Histamin signifikant wirksamer (4:1) als das C-Analogon.

Toxizitãtsuntersuchungen von B 1 und B 2 nach intraperitonealer Applikation an Mäusen ergaben praktisch gleiche $L D_{50}$-Werte ${ }^{11}$. Auch das Vergiftungsbild beider Substanzen ist gleich. Sie zeigen beide eine deutliche Spättoxizität.

Die untersuchten pharmakologisch-toxikologischen Eigenschaften der beiden Analoga B 1 und B 2 sind also weitgehend gleich. Die pharmakologischen Eigenschaften der Silicium-Verbindungen A 2 und B 2 lassen sich zwanglos in die bestehenden Theorien zur Wechselwirkung zwischen Pharmaka und Rezeptoren einordnen.

In Analogie zu den bereits früher von uns beschriebenen Sila-Antihistaminika unterscheiden sich A 2 und B 2 ebenso wie diese bezüglich ihrer Wirkdauer ganz erheblich von den C-Muttersubstanzen. Auf Grund der Hydrolyselabilität der Si-OC-Gruppierung nimmt die Wirkintensität von A 2 und B 2 mit der Zeit stark ab. Messungen in Normaltyrode bei $\mathrm{pH} 7,4$ und $37^{\circ} \mathrm{C}$ ergaben für A 2 eine Zerfallszeit von 28 Min., für B 2 von etwa 14 Min.

Unser Dank gilt dem Verband der Chemischen Industrie, Frankfurt am Main, für die Unterstützung mit Sachmitteln, der Bayer AG, Leverkusen, für die Überlassung von Chlorsilanen sowie Dr. H.-U. Rossée und Prof. Dr. F. Meyer für viele wertvolle Hinweise und Diskussionen.

\section{Literatur}

1 3. Mitt.: R. Tacke und U. Wannagat, Mh. Chem. 107, 439 (1976).

2 Mit Auszügen aus der Dissertation R. Tacke, Techn. Univ. Braunschweig 1974.

3 N. Brock, D. Lenke und H. H. Abel, Arzneimittel-Forsch. 17, 1005 (1967).

* R. Tacke und $U$. Wannagat, Mh. Chem. 107, 111 (1976).

5 R. Tacke und $U$. Wannagat, Mh. Chem. 106, 1005 (1975).

6 H. Arnold, N. Brock und E. Kühas, Asta-Werke AG, D.A.S. 1052982 (29. 6. 1957); Chem. Zentr. 1961, 5182.

' G. H. Wagner, D. L. Bailey, A. N. Pines, M. L. Dunham und D.B. McIntire, Ind. Engng. Chem. 45, 367 (1953).

8 A.D. Petrov, A. F. Plate, E. A. Chernyshev, M. E. Dolgaya, N. A. Belikova, T. L. Krasnova, L. A. Leites, M. A. Pryanishnikova, G. S. Taits und B.I. Kozyrkin, Zh. Obshch. Khim. 31, 1199 (1961); Chem. Abstr. 55,27131 (1961). 
9 H. G. Kuivila und C. R. Warner, J. Org. Chem. 29, 2845 (1964).

10 R. F. Cunico, J. Org. Chem. 36, 929 (1971).

11 F. Meyer und H.U. Rossée, unveröffentlicht; H.-U. Rossée, Dissertation Technische Universität Braunschweig 1974.

Korrespondenz und Sonderdrucke:

Dr. R. Tacke

Institut für Anorganische Chemie

Technische Universität Braunschweig

Pockelsstraße 4

D.3300 Braunschweig

Bundesrepublik Deutschland 